\title{
Simulation Monte Carlo des dépôts de doses en radiothérapie curiethérapie et déploiement sur grille de calcul
}

\author{
C.O. THIAM ${ }^{1}$, L. MAIGNE ${ }^{1}$, B. HABIB ${ }^{1}$, D. DONNARIEIX ${ }^{2}$, J. ARNOLD ${ }^{1}$, \\ V. BRETON ${ }^{1}$
}

(Manuscrit reçu le 30 juin 2006, accepté le 27 octobre 2006)

RÉSUMÉ La méthode de calcul Monte Carlo est reconnue aujourd'hui comme l'algorithme pouvant modéliser au plus près les phénomènes physiques liés aux dépôts d'énergie dans un milieu. D'où l'intérêt d'utiliser cette méthode dans la planification de traitement du cancer par rayonnement, les systèmes de planification traitement (TPS) existant étant limités dans la précision des calculs pour certains cas bien spécifiques. Dans cette approche, nous nous intéressons à la validation du code de calcul Monte Carlo GATE pour les applications dosimétriques en physique médicale. Cependant, l'utilisation de la méthode Monte Carlo pour des géométries complexes ou des images de haute résolution nécessite de nombreuses heures de calculs. En effet, un résultat précis ne peut être obtenu qu'en générant beaucoup d'événements. Nous étudions donc les capacités de la grille de calcul de manière à réduire le temps de calcul en déployant nos simulations avec GATE sur un environnement de calcul distribué.

ABSTRACT Monte Carlo simulation of doses deposits for radiotherapy - brachytherapy and deployment on the computing grid.

Monte Carlo is the algorithm that most closely models the actual physics of the energy deposition process. Thus it would be very interesting to use this method in the cancer treatment planning by radiation. Indeed, existing treatment planning systems (TPS) are limited in the accuracy of dose calculations for certain specific cases. In this approach, we are interested in the validation GATE Monte Carlo toolkit for dosimetric applications in medical physics (Radiotherapy and Brachytherapy). However, the use of Monte Carlo method for complex geometries or images with a large resolution requires a large computing time. Indeed, a precise result can be obtained only by generating a large number of events. We thus study the performances of the computing grid to reduce computing time by deploying GATE simulations on the grid architecture developed within the framework of EGEE European project.

Key words: radiotherapy / dose calculation, GATE Monte Carlo simulation / computing grid

\footnotetext{
1 Laboratoire de Physique Corpusculaire, 24 avenue des Landais, 63177 Aubière Cedex, France.

2 Centre Jean Perrin, Unité de Physique Médicale, Département de Radiothérapie-Curiethérapie, 58 rue Montalembert, 63011 Clermont-Ferrand Cedex, France.
} 


\section{Introduction}

L'équipe PCSV (Plate forme de Calcul pour les Sciences du Vivant) du Laboratoire de Physique Corpusculaire (LPC) à Clermont-Ferrand est impliquée dans le déploiement d'applications biomédicales sur un environnement de calcul distribué. Une de ces applications concerne le déploiement de la plateforme de simulation MC GATE (Geant4 Application for Tomographic Emission, http:// www.opengatecollaboration.org) pour les calculs dosimétriques. L'infrastructure de grille utilisée pour notre déploiement est fournie par le projet européen EGEE (Enabling Grids for E-sciencE, http://public.eu-egee.org/) qui s'inscrit dans la continuité du projet DataGrid.

GATE est un outil de simulation dédié à la base pour les applications SPECT (Single Photon Emission Computed Tomography) et PET (Positron Emission Tomography) en médecine nucléaire ; son champ d'application s'étend aussi aux calculs dosimétriques. Nous présentons dans cet article quelques exemples de simulations de traitements de radiothérapie avec GATE.

Le but de nos études est de permettre l'utilisation de la plateforme GATE dans la planification de traitements en routine clinique. Cependant, cette perspective n'est possible que si le temps de calcul est fortement réduit. Nos simulations GATE sont donc parallélisées en plusieurs petites simulations qui sont déployées sur la grille de calcul. Les tests de temps de calcul effectués, montrent un gain pouvant aller jusqu'à 20. Afin de faciliter l'accès sur la grille pour les utilisateurs (physiciens médicaux), nous avons développé toutes les fonctionnalités sur un portail web GENIUS (Grid Enabled web enNvironment for site Independent User job Submission) pour créer, soumettre et gérer des simulations MC GATE sur l'infrastructure de grille EGEE.

\section{La plate forme de simulation Monte Carlo GATE}

\subsection{Description}

GATE est une plate forme de simulation Monte-Carlo s'appuyant sur les nombreuses potentialités offertes par le code GEANT4 (ensemble complet de modèles physiques validés, description de géométries complexes, génération et suivi des particules, visualisation des volumes et des trajectoires des particules; Jan et al., 2004; Lazaro, 2003), auxquelles ont été adjoints des modules spécifiques dédiés aux besoins des applications en médecine. La plate forme se compose actuellement d'environ 200 classes $\mathrm{C}++$ constituant un ensemble modulaire et orienté objet de composants pour le SPECT et le PET et également la dosimétrie. En plus des librairies de GEANT4 qui constituent la base de GATE, 
nous avons aussi des classes définissant ses propres mécanismes, à savoir la gestion du temps, de la géométrie, des sources et du traitement des événements de la simulation (hits). Ceci inclut la construction, le positionnement, la réplication et le mouvement des volumes, ainsi que la prise en charge de la décroissance radioactive de sources multiples. Certaines classes de base sont directement dérivées de classes de GEANT4 tandis que d'autres définissent des hiérarchies de nouvelles classes et des collaborations de classes. Une couche application implémente les classes concrètes dérivées des classes de la couche de base : ce sont par exemples les classes permettant la construction de volumes de forme spécifique (boîte, sphère) ou des opérations sur des volumes (rotation, translation), ou bien des classes assurant le traitement des évènements (modèles de résolution en énergie, ...). Les utilisateurs peuvent étendre les fonctionnalités de GATE en développant de nouvelles classes à l'intérieur de cette couche d'application. Le langage de script GEANT4 a été étendu pour chacune des classes de base et d'application afin de permettre un contrôle interactif des objets. Ce langage de script fait qu'il n'est pas nécessaire de savoir programmer en $\mathrm{C}++$ pour utiliser GATE : la définition de tous les paramètres de la simulation et le contrôle de cette dernière sont réalisés à l'aide de ces scripts ou par l'exécution de macros.

Depuis 4 ans, l'équipe PCSV du LPC de Clermont Ferrand explore l'utilisation de GATE pour une meilleure planification des traitements en radiothérapie et curiethérapie, en collaboration avec Denise Donnarieix, physicienne médicale au centre Jean Perrin de Clermont Ferrand. Plusieurs études ont été menées pour valider GATE. Les résultats de certains de ces études sont présentés dans le paragraphe suivant.

\subsection{Validation de la plate forme de simulation Monte Carlo GATE}

\subsection{1. Études dosimétriques des électrons dans les tissus}

\subsubsection{Simulations des sources $\beta^{-}$utilisées en curiethérapie}

En routine clinique, la curiethérapie oculaire est utilisée pour traiter certains cancers (les mélanomes de la choroïde par exemple). La dose est délivrée par des disques ophtalmiques radioactifs de $\mathrm{Ru}^{106} / \mathrm{Rh}^{106}$ ou I ${ }^{125}$ (Fig. 2), que l'on fixe directement sur l'œil, au niveau de la tumeur (Maigne, 2005). Cette dose délivrée est très élevée (de l'ordre de $400 \mathrm{~Gy}$ ) et est localisée à la base de la tumeur. Le $\mathrm{Ru}^{106}$ est un émetteur $\beta^{-}$de période 373 jours. Il se désintègre en $\mathrm{Rh}^{106}$ avec une énergie maximale des particules $\beta$ de $39 \mathrm{keV}$. Le parcours des particules $\beta$ issues de la désintégration étant très petit, on considère donc qu'elles sont entièrement absorbées par la fenêtre d'argent des applicateurs. La contribution primaire à la dose thérapeutique provient des particules $\beta$ issues de la désintégration du $\mathrm{Rh}^{106}$. 




Figure 1 - Schéma de décroissance du $R h^{106}$. Decay scheme of ${ }^{106}$ Rh nuclide.

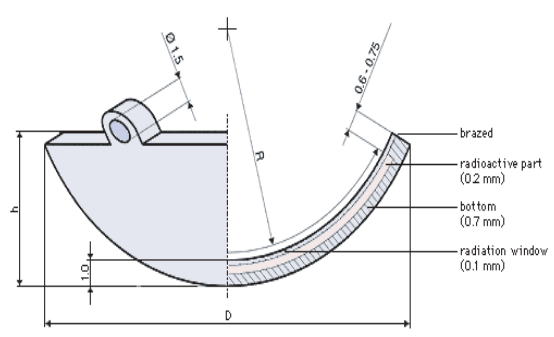

Figure 2 - Applicateur ophtalmique. Scheme of an ophthalmic applicator.

Celui-ci se désintègre en $\mathrm{Pd}^{106}$ (élément stable) avec une période de 29,8 secondes et une énergie maximale de 3,54 MeV. Le schéma de décroissance du $\mathrm{Rh}^{106}$ est représenté sur la figure 1 .

L'énergie moyenne est de 1,41 MeV. Cette désintégration $\beta^{-}$est accompagnée de raies $\gamma$ avec trois raies principales d'énergie $511,86 \mathrm{keV}(21 \%), 621,84 \mathrm{keV}$ $(9,95 \%)$ et $1050,34 \mathrm{keV}(1,47 \%)$.

Nous avons simulés avec GATE les dépôts de doses issus de quelques modèles d'applicateurs contenant des sources de $\mathrm{Ru}^{106} / \mathrm{Rh}^{106}$ (Maigne, 2005 ; Maigne et al., 2004 ; Masurier, 1999 ; Mobit et Badragan, 2004 ; Nath et al., 1995 ; Soares et al., 2001 ; Tjho-Heslinga et al., 1999). Les résultats obtenus avec la version 1.2.0 de GATE (adaptée à geant4.6.2.p02) ont été comparés aux calculs théoriques de la méthode de Hokkanen (Hokkanen et al., 1997) et du logiciel analytique Plaque Simulator (http://radonc.usc.edu/USCRadOnc/Downloadable/PlaqueSimulator.html/) utilisé au centre Jean Perrin de Clermont-Ferrand. Nous avons aussi comparé les résultats de GATE aux mesures obtenues avec des scintillateurs en plastique pour l'étalonnage des applicateurs (mesures BEBIG, http://www. bebig.de/downloads/fs_aug-a_e.pdf/).

Les incertitudes assignées à ces mesures sont évaluées à $\pm 20 \%$. La figure 3 représente les comparaisons de doses pour les applicateurs CCA, CCB. Ces comparaisons donnent de bons accords en particulier pour des distances supérieures à $1,5 \mathrm{~mm}$.

Les résultats obtenus à ce jour dans un milieu homogène sont très encourageants. La comparaison des simulations Monte Carlo GATE et des mesures expérimentales publiées (NIST; Soares et al., 2001) concernant la distribution de la dose sur l'axe du disque ophtalmique CCB donne des différences inférieures à 5,7\% entre 1 et $12 \mathrm{~mm}$ de profondeur (Fig. 4). 


\section{Plaque Ophtalmique CCA}

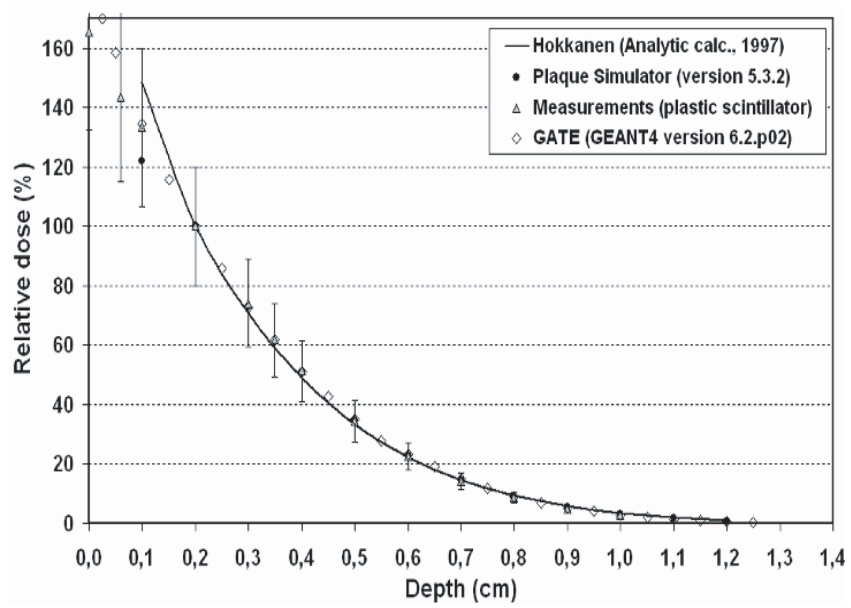

Plaque Ophtalmique CCB

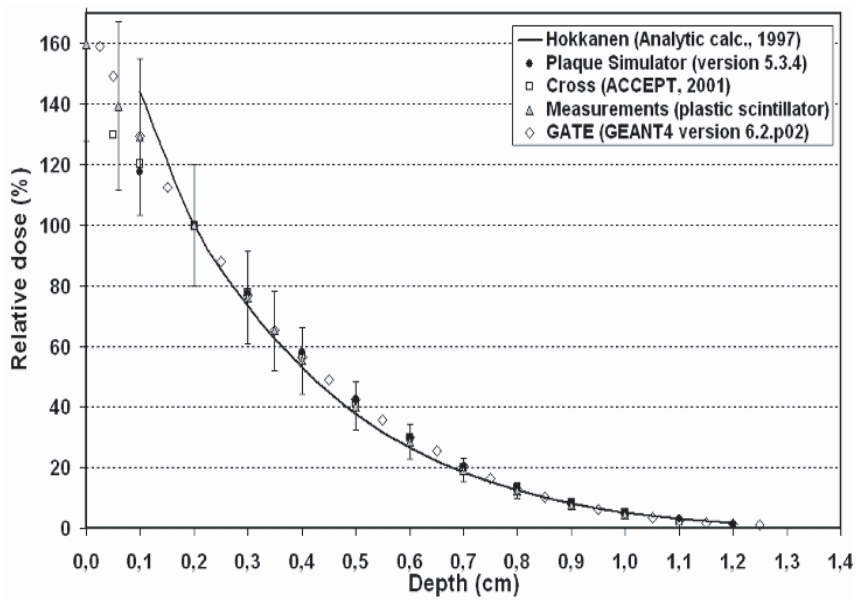

Figure 3 - Comparaison des doses relatives sur l'axe d'un applicateur dans l'eau (doses normalisées à la valeur axiale et à une profondeur de $2 \mathrm{~mm}$ ).

Comparison of calculated and measured relative doses on the axis of a $\mathrm{CCA}$ and $\mathrm{CCB}$ applicators in water (doses are normalized to the axial value at a depth of $2 \mathrm{~mm}$ ). 


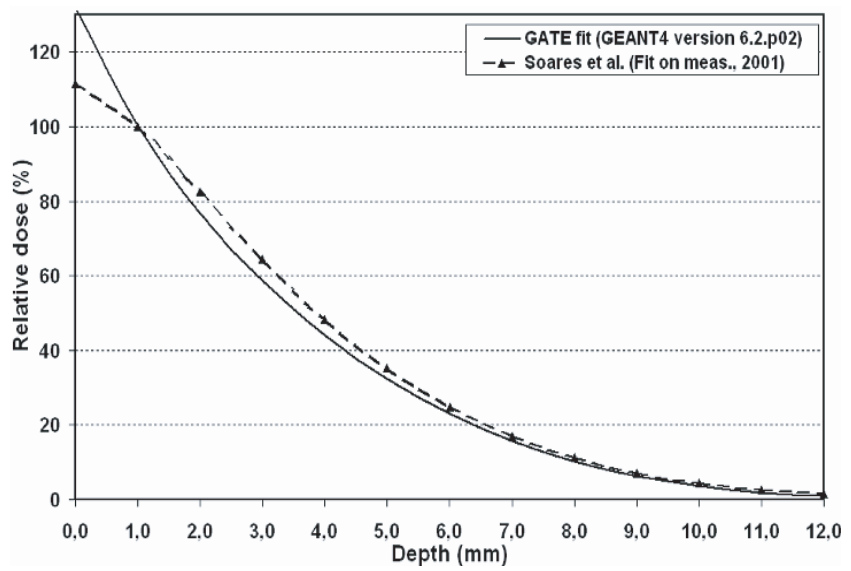

Figure 4 - Comparaison entre GATE et les valeurs moyennes mesurées dans l'eau (Soares et al., 2001). Comparisons of fitted GATE results with average measured values in water (Soares et al., 2001).

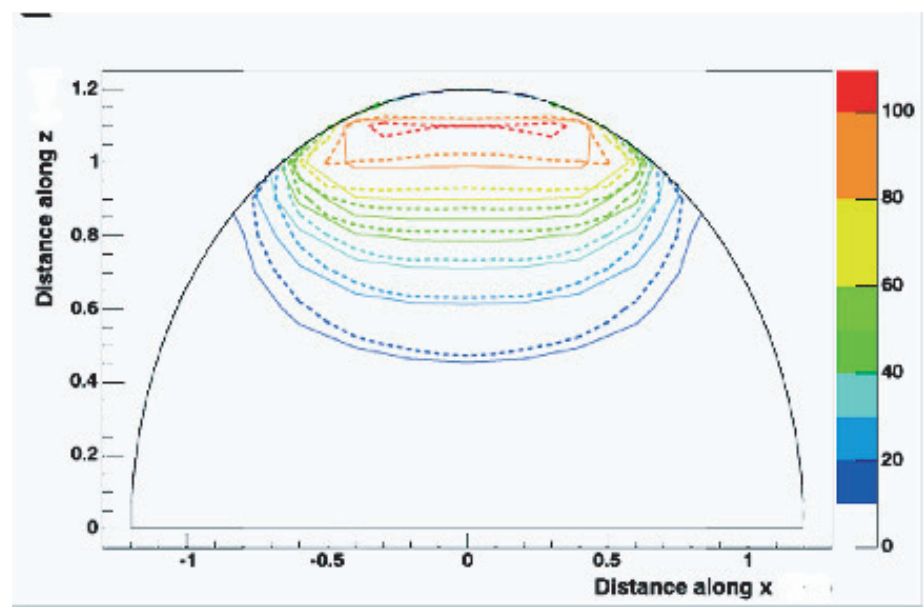

Figure 5 - Comparaison des contours Isodoses dans l'eau pour l'applicateur CCA : doses normalisées à $100 \%$.

Comparison of isodose contours for doses in water from a CCA applicator between GATE calculations and Plaque Simulator analytic calculations. Doses have been normalized to $100 \%$.

La comparaison des simulations avec le logiciel de planification des traitements Plaque Simulator donne un écart de $10 \%$ pour les applicateurs CCA et $\mathrm{CCB}$; ce qui nous conforte dans l'utilisation des valeurs d'étalonnage fournies par BEBIG et dans l'utilisation clinique du logiciel. 
TABLEAU I

Différences relatives entre GATE - « Plaque Simulator » - « Point Kernel ». Relative variations between GATE, Plaque Simulator and Point Kernel methods.

\begin{tabular}{ccccccccc}
\hline & \multicolumn{2}{c}{ CCA } & \multicolumn{2}{c}{ CCB } & \multicolumn{2}{c}{ CCX } & \multicolumn{2}{c}{ CCZ } \\
\cline { 2 - 8 } & $\begin{array}{c}\text { Point } \\
\text { kernel } \\
\text { integration }\end{array}$ & $\begin{array}{c}\text { Plaque } \\
\text { Simulator }\end{array}$ & $\begin{array}{c}\text { Point } \\
\text { kernel } \\
\text { integration }\end{array}$ & $\begin{array}{c}\text { Plaque } \\
\text { Simulator }\end{array}$ & $\begin{array}{c}\text { Point } \\
\text { kernel } \\
\text { integration }\end{array}$ & $\begin{array}{c}\text { Plaque } \\
\text { Simulator }\end{array}$ & $\begin{array}{c}\text { Point } \\
\text { kernel } \\
\text { integration }\end{array}$ & $\begin{array}{c}\text { Plaque } \\
\text { Simulator }\end{array}$ \\
\hline $2 \mathrm{~mm}$ off & $9,1 \%$ & $10,0 \%$ & $14,4 \%$ & $9,5 \%$ & $0,5 \%$ & $6,1 \%$ & $4,6 \%$ & $9,3 \%$ \\
$4 \mathrm{~mm}$ off & $2,1 \%$ & $9,6 \%$ & $11,8 \%$ & $9,1 \%$ & $26,4 \%$ & $10,3 \%$ & $34,3 \%$ & $27,5 \%$ \\
\hline
\end{tabular}

La forme des isodoses avec plaque simulator concorde bien avec les calculs de GATE (Fig. 5 ; Maigne, 2005). Pour mieux estimer les quelques différences observées entre GATE et les autres méthodes, nous comparons dans le tableau I les doses à $2 \mathrm{~mm}$ et $4 \mathrm{~mm}$ de l'axe central et à $3 \mathrm{~mm}$ de profondeur, ce qui représente typiquement la distance approximative entre le centre d'un applicateur ophtalmique et la distance thérapeutique pour un mélanome de l'œil de taille et d'épaisseur moyenne par exemple. Nous pouvons remarquer que les résultats avec la méthode du point kernel (Douglas et al., 1990) sont en bon accord avec GATE auprès de l'axe central $(2 \mathrm{~mm})$ par rapport à «plaque simulator », excepté le modèle CCX. Mais pour des distances plus grandes par rapport à l'axe central la méthode du point kernel donne des différences importantes par comparaison à notre calcul Monte Carlo, particulièrement pour de petites plaques CCX et CCZ.

En résumé, l'étude des distributions de dose calculées avec GATE pour les applicateurs ophtalmiques a montré des résultats relativement satisfaisants par rapport aux autres méthodes (soit par Monte Carlo soit par calculs analytiques ; Maigne et al., 2004).

\subsubsection{Simulation des faisceaux d'électrons utilisés en radiothérapie}

En radiothérapie externe, il est indispensable d'avoir une connaissance précise de la dose délivrée dans le volume cible et dans les organes à risques avoisinants. Pour être utilisables cliniquement, les modèles de calcul doivent tenir compte des caractéristiques exactes des faisceaux utilisés et des densités des tissus. Nous avons simulés avec GATE les dépôts de doses dans l'eau des faisceaux d'électrons issus de la tête d'irradiation de l'accélérateur SL-ELEKTA20 (Bramoullé, 2000) utilisé pour des traitements de radiothérapie au centre Jean Perrin de Clermont Ferrand.

La tête d'irradiation de l'accélérateur contient le système de mise en forme du faisceau en vue de son utilisation thérapeutique. Sa description précise est cruciale pour les calculs de dose. Elle fixe le mode de fonctionnement de l'accélérateur 


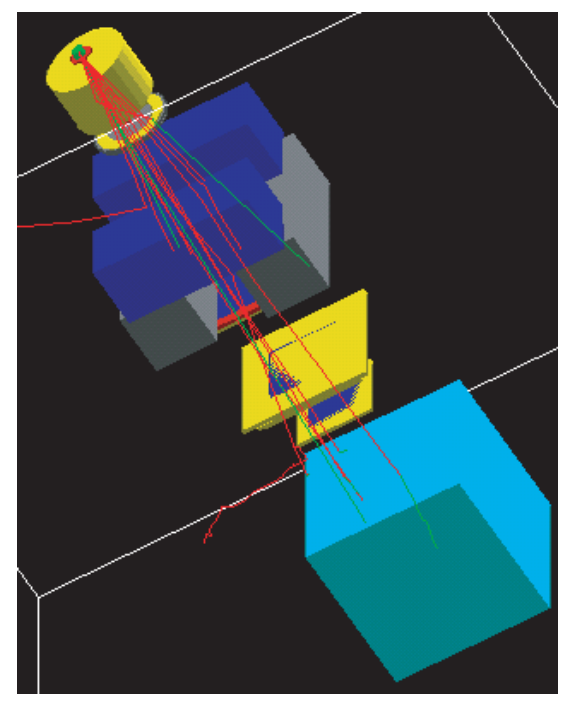

Figure 6 - Modélisation 3D de la tête d'irradiation dans GATE.

Modelling 3D of the irradiation head.



Figure 7 - Distribution de la dose dans l'eau avec un champ d'irradiation de $10 \times 10 \mathrm{~cm}^{2}$. Dose distribution in water with irradiation field of $10 \times 10 \mathrm{~cm}^{2}$.

(Electron ou Photon) (Bramoullé, 2000 ; Douglas et al., 1990 ; Hedtjarn et al., 2000 ; Hokkanen et al., 1997 ; Jan et al., 2004 ; Lazaro, 2003 ; Maigne, 2005 ; Maigne et al., 2004 ; Masurier, 1999) et permet de contrôler les caractéristiques du faisceau (dimension, intensité,...). La modélisation de cette partie de l'accélérateur par GATE est effectuée précisément suivant les données du constructeur. Tous les éléments caractérisant les faisceaux d'électrons sont pris en compte. La figure 6 donne une visualisation en 3D de la tête modélisée en mode électrons.

La figure 7 représente une comparaison entre la distribution de la dose dans une cuve d'eau $\left(30 \times 30 \times 30 \mathrm{~cm}^{3}\right)$ obtenue par GATE et les mesures expérimentales réalisées au centre Jean Perrin. La courbe représente la variation de dose absorbée dans un plan perpendiculaire à l'axe du faisceau et à une profondeur de référence de 12,5 mm. Cette courbe donne l'une des caractéristiques les plus importantes définissant l'efficacité et la précision du faisceau thérapeutique. Tous les processus physiques ont été simulés. On observe donc un bon accord entre les calculs de GATE et les mesures du Centre Jean Perrin (CJP) à l'exception des bordures du champ d'irradiation. Ce désaccord est sans doute lié à la complexité 
SIMULATION MONTE CARLO DES DÉPÔTS DE DOSES

de certains éléments de la géométrie (systèmes de collimations). Des corrections sont à l'étude pour améliorer les premiers résultats obtenus.

\subsection{2. Études dosimétriques des photons : simulations des sources $\gamma$ utilisées en curiethérapie}

Dans la lutte contre le cancer, certains traitements de curiethérapie à bas débit de dose utilisent des sources cylindriques (grains $\mathrm{I}^{125}$; Hedtjarn et al., 2000). Ce type de radioélément est caractérisé par une désintégration de capture électronique de laquelle émanent des rayonnements $X$ et gamma de faible énergie (de l'ordre de $30 \mathrm{keV}$ ) entraînant une dose au contact de la source élevée et une diminution rapide de celle-ci avec la profondeur. Dans cette étude, nous utilisons GATE pour modéliser des sources cylindriques d' ${ }^{125}$ utilisées en curiethérapie oculaire et prostatique. Cette étude permettra à terme de valider GATE pour les faibles énergies.

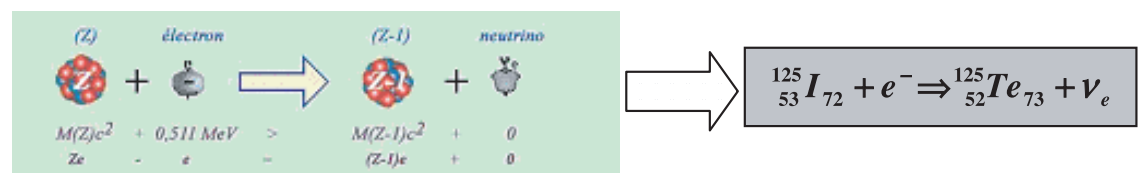

Figure 8-Schéma de décroissance $I^{125}$ par capture électronique. Decay scheme of ${ }^{125}$ I nuclide by electronic capitation.

Depuis 1972, le « N.I.S.T. » (National Institute of Standards and Technology) a connu des avancées importantes en termes de calibration des sources $\mathrm{I}^{125}$ et de définition d'un formalisme permettant de déterminer la dose dans l'eau. Pour effectuer nos études dosimétriques, nous nous sommes référés aux travaux du groupe de travail « Task Group 43 » de l'American Association of Physicists in Medecine (A.A.P.M.) (Mobit et Badragan, 2004 ; Nath et al., 1995) datant de 1995 et revu en 2004, donnant une estimation de la constante $\Lambda$ (constante de débit de dose) et un formalisme de calcul de la dose dans l'eau. L'expression du débit de dose en un point $P(r, \theta)$ (Fig. 9) est la suivante :

$$
\dot{D}_{\text {eau, eau }}(r, \theta)=S_{K} \Lambda \frac{G(r, \theta)}{G\left(r_{0}, \theta_{0}\right)} g(r) F(r, \theta) .
$$

Dans cette expression, $S_{K}$ définit le kerma dans l'air « air kerma strength » en un point $P$ situé sur l'axe transverse de la source $\left(\theta_{0}=\pi / 2\right)$

$$
S_{K}=\dot{K}_{a i r, a i r}\left(r, \frac{\pi}{2}\right) r^{2}
$$




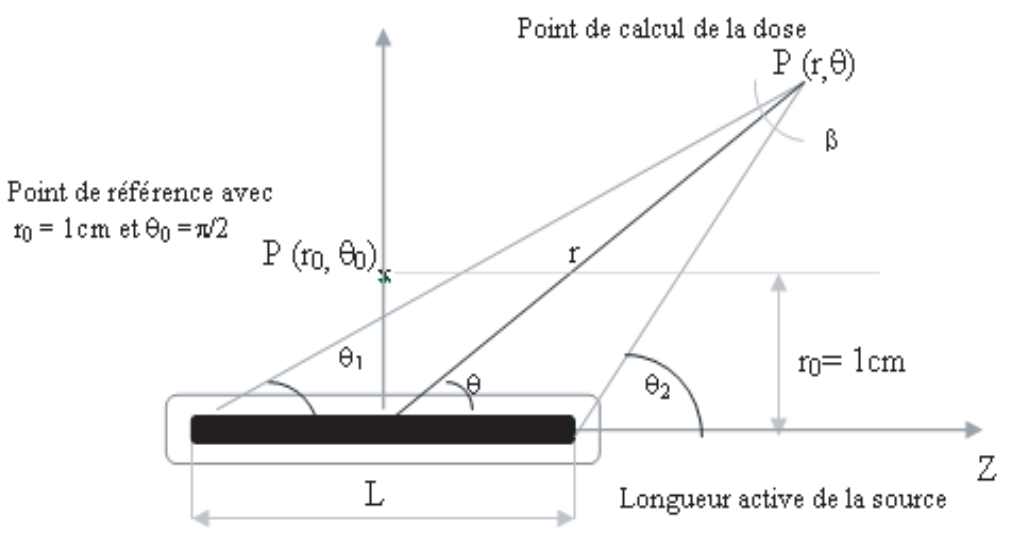

Figure 9 - Système de coordonnées polaires utilisé pour des calculs dosimétriques en curiethérapie. Coordinate system used for brachytherapy dosimetry calculation.

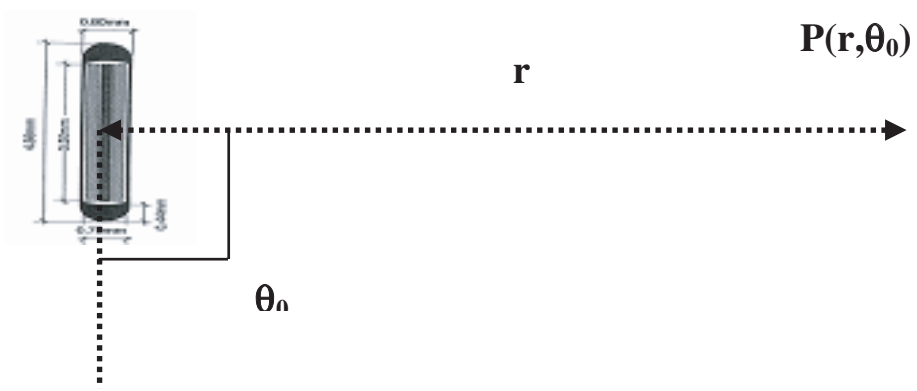

Figure 10 - Mesure du débit de kerma dans l'air, $\dot{K}_{\text {air, air }}(r, \pi / 2)$.

Air kerma strength estimation method.

Le débit de kerma dans l'air, $\dot{K}_{a i r, \text { air }}(r, \pi / 2)$, variant en $1 / d^{2}$ pour une source ponctuelle, la quantité $S_{K}$ devient indépendante de la distance du point de mesure à la source. Elle est ainsi représentative de l'activité de la source.

Le paramètre $g(r)$ permet de tenir compte des effets d'absorption et de diffusion dans le milieu situé entre le point $P_{0}$ de référence et le point $P(r, \theta)$.

La constante de débit de dose (débit de dose dans l'eau à $1 \mathrm{~cm}$ sur l'axe de la source pour une unité de kerma strength) est définit par et son expression est donnée par l'équation suivante :

$$
\Lambda=\frac{\dot{D}\left(r_{0}=1 \mathrm{~cm}, \theta_{0}=\pi / 2\right)}{S_{K}}
$$


$G(r, \theta)$ est un facteur géométrique traduisant le phénomène d'atténuation de la fluence des photons dans le milieu

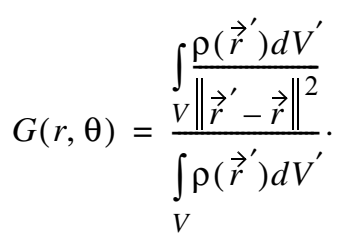

Il permet de tenir compte de la diminution de la fluence des photons en fonction de la distance à la source. Autrement dit, elle calcule le nombre de photons qui atteignent un point du milieu à partir du nombre initial de photons en un point de départ, dit de référence. Pour une approximation ponctuelle de la source, cette diminution se fait en $1 / r^{2}$. Pour une approximation linéaire de la source, l'expression de $G(r, \theta)$ devient la suivante :

$$
G(r, \theta)=\left\{\begin{array}{l}
\frac{\theta_{2}-\theta_{1}}{L r \sin \theta} \text { pour } \theta \neq 0^{\circ} \\
\left(r^{2}-\frac{L^{2}}{4}\right)^{-1} \text { pour } \theta \neq 0^{\circ}
\end{array} .\right.
$$

Le paramètre $g(r)$ permet de tenir compte des effets d'absorption et de diffusion dans le milieu situé entre le point $P_{0}$ de référence et le point $P(r, \theta)$

$$
g(r)=\frac{[D(r, \pi / 2) / G(r, \pi / 2)]}{\left[D\left(r_{0}, \pi / 2\right) / G\left(r_{0}, \pi / 2\right)\right]} .
$$

Le paramètre $F(r, \theta)$ définit la fonction d'anisotropie, elle exprime le caractère non ponctuel de la source et des matériaux intervenant dans sa fabrication et traduit les phénomènes d'absorption et de diffusion dans le milieu ainsi que l'encapsulation de la source

$$
F(r, \theta)=\frac{[D(r, \theta) / G(r, \theta)]}{[D(r, \pi / 2) / G(r, \pi / 2)]} .
$$

Trois modèles de sources $\mathrm{I}^{125}$ ont été modélisés avec GATE : le modèle 2301 de B.M.I. (Best Medical International), le modèle Symmetra de UroMed/Bebig et le modèle 6711 d'Amersham.

Les raies énergétiques issues de la désintégration par capture électronique de l'I $^{125}$ ont été simulées selon les recommandations de l'A.A.P.M. (Mobit et Badragan, 2004 ; Nath et al., 1995). 
Fonction de dose radiale : Comparaisons

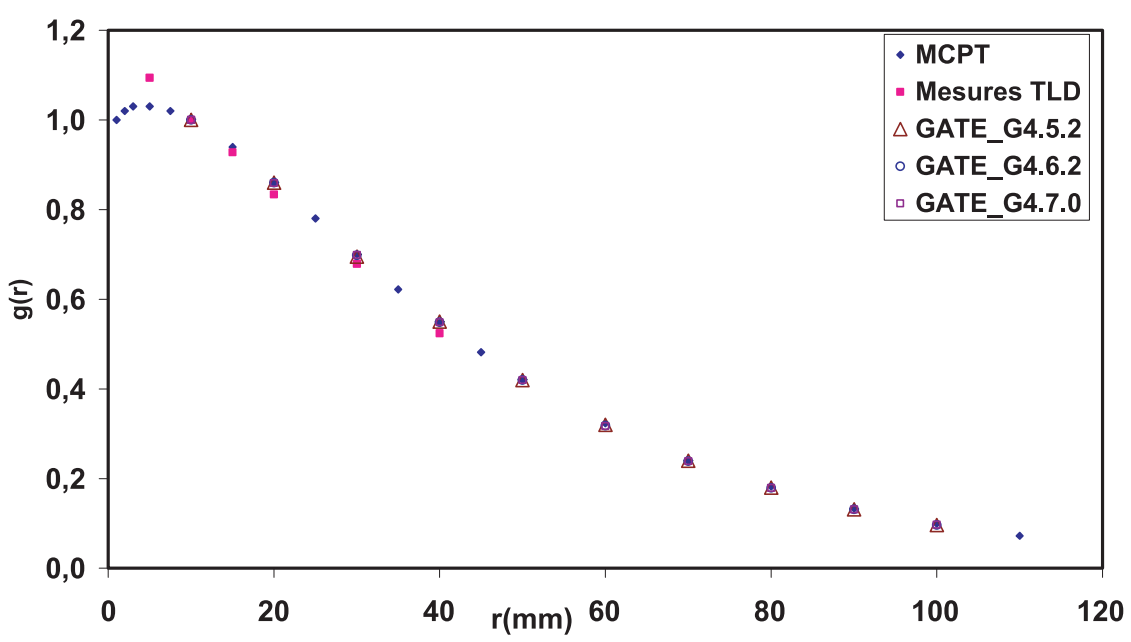

Figure 11 - Représentation graphique des g(r) pour la source Symmetra de Bebig avec 1476000 évènements.

Radial dose function $g(r)$ for Symmetra ${ }^{125}$ I source in liquid water with 1476000 events: comparisons.

Nous présentations ici les résultats obtenus uniquement pour le modèle Symmetra. Les deux autres modèles sont en cours de validation.

La figure 11 représente la fonction de dose radiale obtenue avec différentes versions de GATE dans le cas du modèle Symmetra de UroMed/Bebig. La distribution de doses est calculée sur l'axe transverse de la source pour des distances de 1 à $10 \mathrm{~cm}$ de son centre dans l'eau liquide.

La figure 12 représente la fonction d'anisotropie obtenue pour le même modèle de source dans les mêmes conditions de simulation. Cette grandeur permet de tenir compte dans la détermination de la dose dans l'eau, des phénomènes d'absorption et de diffusion dans le milieu ainsi que de l'encapsulation de la source.

Nous obtenons une bonne corrélation entre les valeurs calculées avec GATE et celles obtenues par le code MCPT (Hedtjarn et al., 2000). L'écart relatif moyen est de l'ordre de $1,3 \%$ dans le calcul de la fonction d'anisotropie à $2 \mathrm{~cm}$ de la source.

La comparaison des mesures expérimentales TLD semblent en bon accort également avec GATE avec un écart relatif moyen de l'ordre de 2,2\%. Le calcul Monte Carlo avec GATE dans le cas de la fonction de doses radiales $g(r)$ montrent 


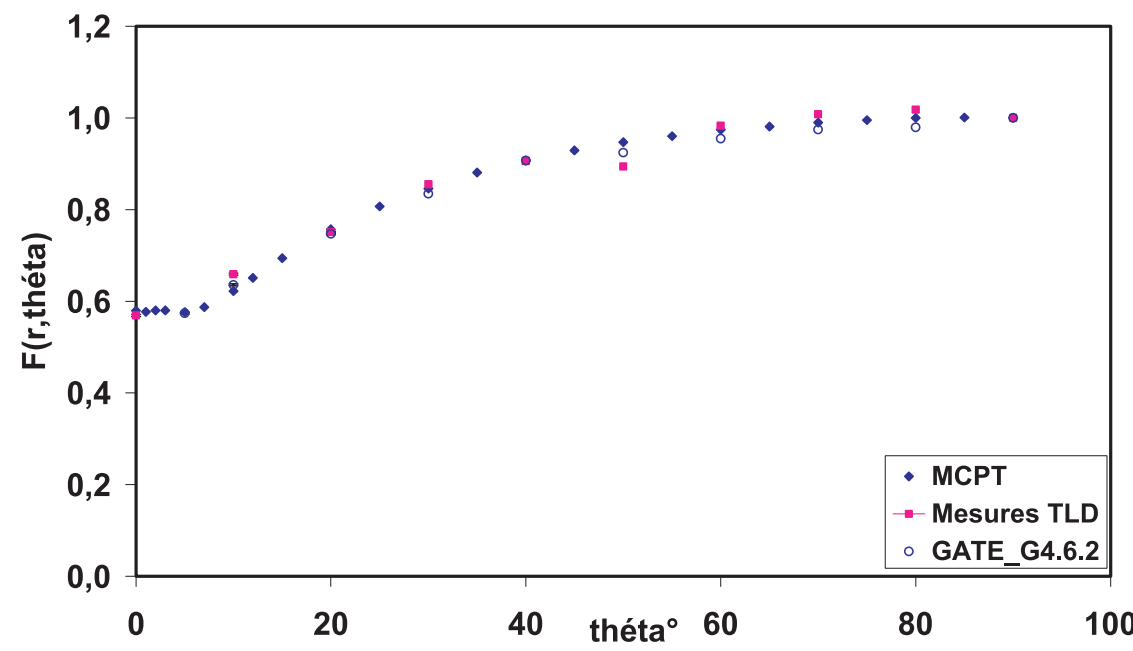

Figure 12 - Représentation graphique des fonctions d'anisotropie à $r=20$ mm pour la source Symmetra de UroMed/Bebig.

Anisotropy function $F(r, \theta)$ for $r=20 \mathrm{~mm}$, Symmetra ${ }^{125}$ I source: comparisons.

\section{TABLEAU II}

Valeurs de la constante de débit de dose en $\mathrm{cGyh}^{-1} \mathrm{U}^{-1}$ obtenue pour le modèle Symmetra. Dose rate constant $\left(\mathrm{cGyh}^{-1} \mathrm{U}^{-1}\right)$ values for Symmetra ${ }^{125}$ I source.

\begin{tabular}{cccccc}
\hline $\begin{array}{c}\text { Activité } \\
\text { de source }\end{array}$ & GATE_G4.5.2 & GATE_G4.6.2 & GATE_G4.7.0 & $\begin{array}{c}\text { Hedjarn } \text { et al., 2000 } \\
\text { MCPT DLC99 }\end{array}$ & $\begin{array}{c}\text { Hedjarn } \text { et al., 2000 } \\
\text { MCPT DLC99 }\end{array}$ \\
\hline $\mathbf{1 . 4 7 6} \mathbf{~ M B q}$ & $0.955 \pm 0.020$ & $0.900 \pm 0.020$ & $1.044 \pm 0.020$ & 1.002 & 1.010 \\
$\mathbf{1 4 . 7 6} \mathbf{~ M B q}$ & $1.050 \pm 0.001$ & $1.010 \pm 0.001$ & $1.000 \pm 0.001$ & 1.002 & 1.010 \\
\hline
\end{tabular}

également une bonne compatibilité entre les trois versions utilisées dans cette étude et un bon accord par rapport au code M.C.P.T. et aux mesures par TLD avec des écarts relatifs inférieurs à $2 \%$.

Ces résultats permettent donc de calculer la constante de débit de dose (débit de dose dans l'eau à $1 \mathrm{~cm}$ sur l'axe de la source pour une unité de «kerma strength ») qui est définie par l'équation (3). Les valeurs calculées avec GATE en comparaison avec les résultats du code M.C.P.T. sont reportées dans le tableau II.

\section{La grille de calcul EGEE}

L'une des perspectives offertes par l'utilisation de GATE en dosimétrie réside dans la possibilité de calculer les dépôts de dose dans les tissus humains modélisés 
à partir d'images obtenues par des imageurs : scanneur, IRM, TEP. Du faite de la très haute résolution de ces images, les dépôts de doses ne sont pas aujourd'hui calculés par des générateurs Monte Carlo, mais de façon analytique. Les grilles de calcul offrent des perspectives très prometteuses pour réduire ces temps de calculs et cette activité de recherche s'est donc inscrit dans le cadre du projet EGEE.

\subsection{Présentation de la grille de calcul EGEE}

Le projet EGEE (Enabling Grids for E-science in Europe ; http://public.euegee.org/) fournit une plate forme de production pour la recherche répartie sur plusieurs sites et impliquant plusieurs communautés d'utilisateurs organisés en groupes de travail. L'accès aux ressources de la grille nécessite donc pour l'utilisateur, d'être affilié à une organisation virtuelle (VO). Dans le cas de l'application GATE, il faut être membre de la VO «biomed». Un certificat d'autorisation, numérique et associé à des mots de passe, est attribué à tous les utilisateurs afin de garantir un accès sécurisé aux ressources de la grille.

L'utilisateur interagit avec la grille via une interface utilisateur (UI) lui permettant :

- d'interroger le système d'information pour connaître les ressources disponibles,

- de manipuler des données : les déposer sur des unités de stockage (SE), les enregistrer sur des catalogues, les dupliquer...

- de soumettre des travaux (jobs).

Les sites mettant à disposition des ressources possèdent un ou plusieurs des éléments suivants :

- UI (User Interface): interface utilisateur, machine depuis laquelle un utilisateur soumet un job,

- CE (Computing Element) : élément de calcul comprenant un ou plusieurs nœuds de calcul (Worker Nodes WN) et un frontal qui assure la correspondance entre un utilisateur de la grille et un compte disponible sur un CE pour la VO de l'utilisateur (GateKeeper GK),

- SE (Storage Element) : élément de stockage (serveur de disques),

- MON (Monitoring Node) : nœud de contrôle, machine chargée de faire tourner les fonctionnalités du système d'information pour le site et le service d'optimisation des duplications de fichiers,

- RB (Resource Broker) : courtier de ressources, machine qui recherche sur la grille l'élément de calcul le plus adéquat pour l'exécution du job en prenant en compte la proximité des données éventuellement requises. 
Des services de maintenance des catalogues (localisations des fichiers originaux et leurs copies), d'information (R-GMA) (état de la grille, infrastructure et applications) et de gestion (événements relatifs à la vie d'un job) sont aussi disponibles.

Le but du système d'information de la grille est de permettre la gestion et la surveillance des ressources distribuées. La plate forme de simulation Monte Carlo GATE est disponible sur plusieurs sites EGEE. Un ensemble de commandes développées dans le middleware EGEE permet de soumettre et de gérer des jobs sur la grille. L'utilisateur a la possibilité d'enregistrer et de gérer les données (Images médicales, logiciels, ...) et d'accéder aux unités de calculs à travers différents sites dans le monde. Il est possible d'évaluer les ressources de travail pour la VO à laquelle on appartient (temps CPU, utilisation mémoire, ...).

\subsection{Déploiement de GATE sur la grille EGEE}

Les simulations Monte Carlo utilisent des générateurs de nombres pseudo aléatoires de manière à reproduire la probabilité des interactions physiques dans la matière. Plus les interactions dans la matière sont nombreuses, plus les simulations utilisent un nombre important de nombres aléatoires. Le meilleur moyen pour réduire les temps de calcul incombant aux simulations Monte Carlo est de les subdiviser en un nombre de simulations générant moins de particules. À chacune de ces petites simulations est indexée une sous séquence de nombres pseudo aléatoires obtenue en « découpant » une très longue séquence de nombres, chaque sous séquence de nombre devant être indépendante (Maigne et al., 2004). Nous avons utilisé une méthode de segmentation pour découper la séquence de nombres aléatoires. À chaque sous séquence obtenue, correspond un «état » du générateur aléatoire stocké sous forme d'un fichier. Un job GATE générant des millions d'événements est ainsi parallélisé en plusieurs petites simulations (jobs) qui sont déployées sur la grille de calcul, chacune utilisant un «état» du générateur aléatoire.

La soumission d'un job se fait depuis une interface utilisateur (UI) selon la figure 13 en envoyant au courtier de ressources (Resource Broker) un script JDL (Job Description Language) décrivant les caractéristiques du job sous forme d'attributs. Ces attributs décrivent les ressources nécessaires (CPU, mémoire, logiciel GATE, ...) et les fichiers nécessaires (GateMaterials.db : fichier contenant la description des matériaux utilisés dans la définition de la géométrie dans la simulation...). Un ensemble de commandes développées dans le middleware EGEE permet à l'utilisateur de surveiller ses travaux sur la grille (soumission du job, vérification de l'état du job enregistrement et réplication de données récupération de résultats...). 
Une fois le job soumis, il passe par différents états (Submitted, Waiting, Ready, Scheduled, Running, Done).

\subsection{Optimisation des temps de calcul}

Nous avons effectué des tests sur deux sites EGEE (CC-IN2P3 Lyon et LPC Clermont) sur lesquels l'application GATE est déployée afin d'estimer les temps de calcul. Une simulation GATE générant plusieurs millions d'événements (20 000000 particules) a été divisée successivement en des séries de dix, vingt, cinquante et cent tâches qui ont ensuite été déployées sur la grille de calcul. Nous avons évalué les temps relatifs à la vie de chaque tâche pour chaque série de test. Les résultats présentés sur la figure 14 représentent une moyenne des temps pour chaque cas.

Ces résultats quantifient le temps pour chaque état du job au cours de sa vie sur la grille. Une fois que la requête de soumission est adressée au courtier de ressources $(\mathrm{RB})$, la tâche est dans l'état « submitted ».

- L'état «waiting »: la tâche est en attente pendant que le RB interroge le système d'information pour trouver l'élément de calcul le plus approprié pour accueillir la tâche.

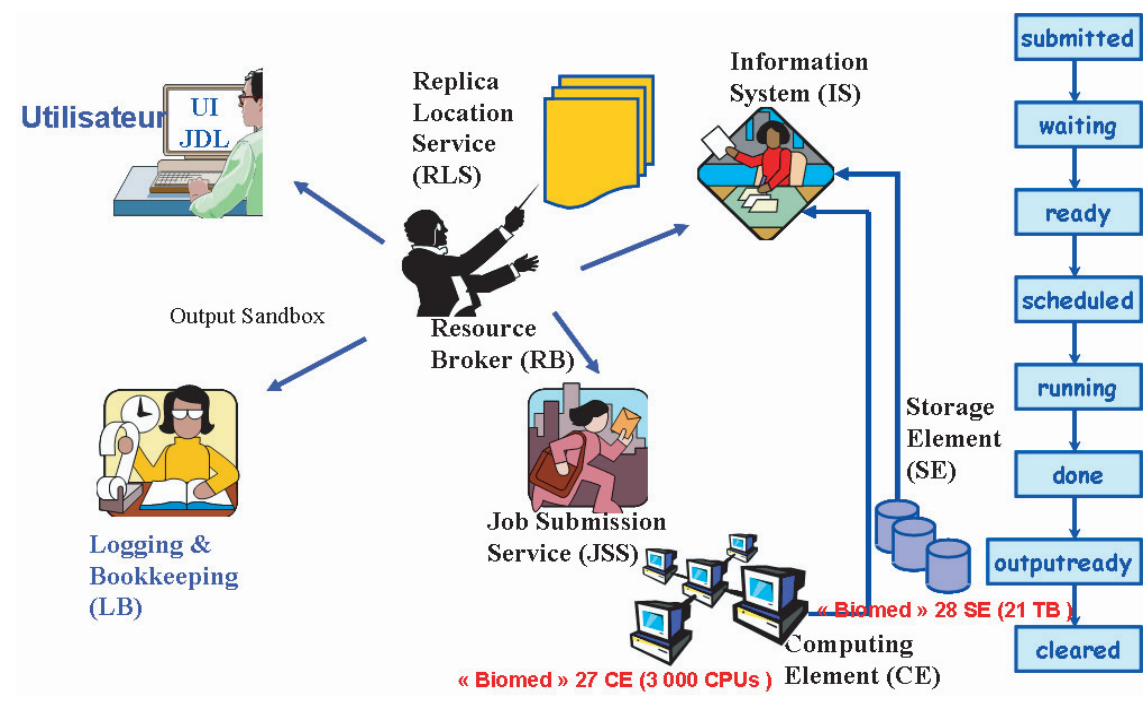

Figure 13 - Schéma d'utilisation de l'infrastructure de grille EGEE.

Schematic use of EGEE computing grid infrastructure. 


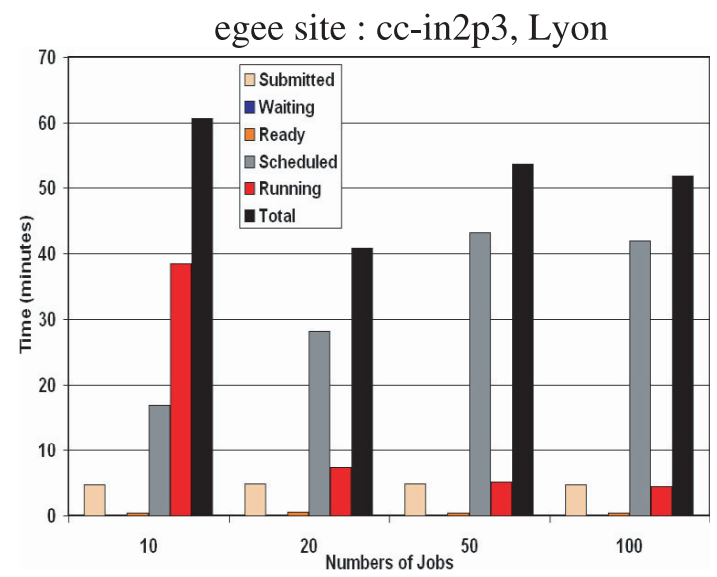

egee site : lpc, Clermont

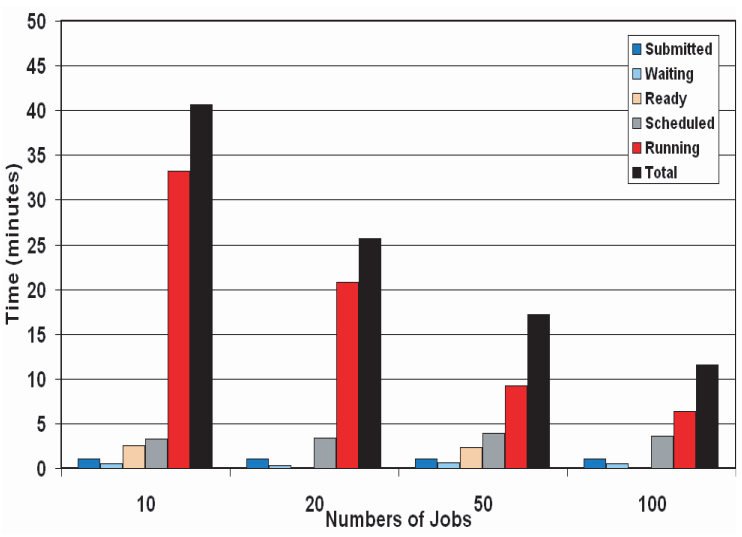

Figure 14 - Comparaison de temps pour différents états des jobs sur la grille.

Computing times comparison for different states of jobs on the grid.

- L'état «Ready»: la tâche est préparée pour être soumise au «Computing Element » (CE).

- L'état «Scheduled» : la tâche est en queue de batch sur le CE.

- L'état « Running »: la tâche tourne.

- L'état « Done »: l'exécution sur le CE est terminée et s'est bien déroulée; le résultat peut être rapatrié à l'utilisateur. Cet état correspond au temps total passé sur la grille pour job. 
egee site : cc-in2p3, Lyon

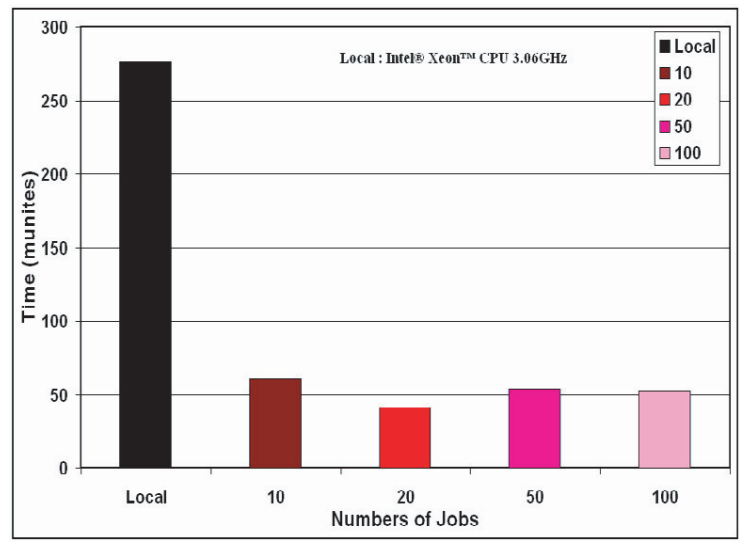

egee site : lpc, Clermont

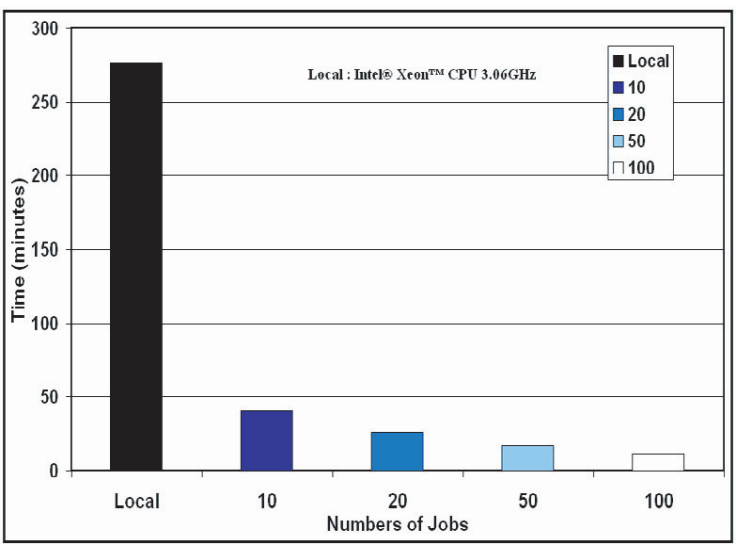

Figure 15 - Comparaison des temps de calcul sur la grille rapport au temps en local. Comparison of total computing times between grid and local computing.

Le temps correspondant à l'état « Scheduled» sur la figure 14 peut être important, cela dépend directement de la charge du CE et de la performance des machines qui constituent les nœuds. Les autres temps sont négligeables par rapport au temps total que passe le job sur la grille.

La figure 15 représente une comparaison du temps d'exécution sur une machine locale de la simulation subdivisée et des jobs déployés sur la grille par rapport aux temps totaux pour chaque série de test. 
Dans le cadre de cette application, nous obtenons un gain très significatif en parallélisant une simulation GATE dont le temps de calcul est de l'ordre de 12 heures sur la grille informatique. Ces tests de temps donnent un gain maximum de 30 pour une division en 100 jobs sur le site EGEE LPC de Clermont. Par contre sur le site CC-IN2P3 Lyon le gain maximum est de seulement 10 pour une répartition en 20 Jobs.

Ces chiffres montrent que la division de la simulation en un très grand nombre de petites simulations n'est pas forcement la meilleure solution pour paralléliser un job GATE. Il est nécessaire de tenir compte de plusieurs facteurs notamment le nombre de ressources disponibles pour l'application, la charge des machines et leurs performances.

\subsection{Le portail web d'accès à la grille : GENIUS}

Dans le but de faciliter l'utilisation de l'application GATE et des ressources de la grille, un portail web a été développé. Ce portail (GENIUS : https://clrpcsv12. in2p3.fr) intègre toutes les fonctionnalités de la grille de calcul de telle sorte que les lignes de commandes EGEE sont élaborées de façon automatique (Adronico et al., 2003). Ce portail Internet (Fig. 16) permettra donc à un physicien médical de pouvoir utiliser l'application GATE dans les planifications de traitement en

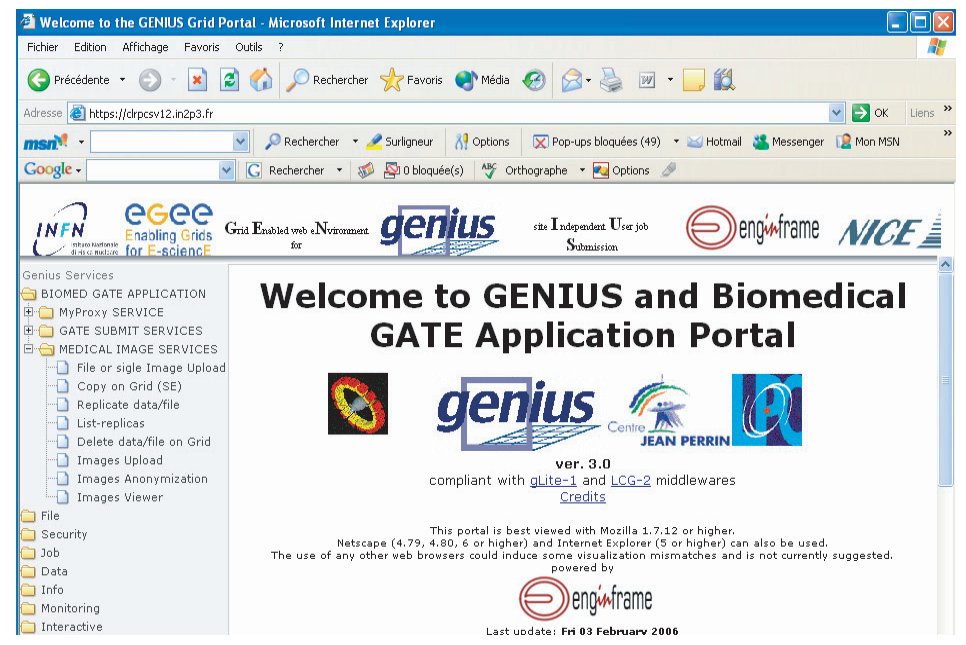

Figure 16 - Portail web d'accès aux ressources de la grille : GENIUS.

Screenshot of the GENIUS web portal. 



Figure 17 - Le Portail GENIUS : pages de création et de gestion de fichier.

Screenshot of the GENIUS web portal to create and manage GATE files.

curiethérapie et radiothérapie, de soumettre des simulations Monte Carlo GATE et d'enregistrer des données sur les ressources de stockage de la grille.

Cependant, les données médicales doivent être anonymisées avant leur enregistrement sur la grille et leur accès via le portail doit être sécurisé et rapide 
(par rapport à la durée de calcul d'une simulation). Ces données (images médicales, DICOM, binaires) peuvent être ensuite utilisées dans les simulations GATE pour des calculs dosimétriques par exemple. Afin d'être sûr que les données médicales anonymisées soient accessibles pour les calculs, leur réplication sur différents éléments de stockage (SE) peut être réalisée grâce aux services de grille qui donnent la possibilité de gérer ces informations de façon libre et transparente (Fig. 17).

Les opérations de manipulation des données et des catalogues sur la grille sont assurées par le système « Replica Manager » qui intègre tous les outils permettant de gérer les données sur la grille, et assure un point d'entrée unique pour les autres utilisateurs et services de la grille.

Les fichiers déposés sont identifiés par un nom logique de fichier LFN (Logical File Name), alias d'un GUID (Grid Unique IDentifier), défini par l'utilisateur.

\section{Conclusion}

Les études menées jusqu'à présent ont permis une utilisation conviviale de GATE avec des simulations Monte Carlo réalistes. Elles ont impliqué la validation de la plate forme GATE utilisant les librairies physiques de GEANT4 pour le calcul de dose absorbée.

Nous avons aussi exploité les fonctionnalités d'une architecture de grille pour distribuer nos simulations Monte Carlo afin d'obtenir un gain significatif en temps de calcul de manière à rivaliser avec les logiciels analytiques actuellement utilisés en centres hospitaliers pour la planification des traitements en radiothérapie.

Les activités autour de l'utilisation de GATE pour la dosimétrie en radiothérapie et curiethérapie sont entrées dans une phase d'évaluation directe avec le centre Jean Perrin de Clermont Ferrand. Un poste de travail est actuellement disponible dans ce centre pour tester l'application GATE sur la grille. Ce service va être simplifié pour en faciliter l'utilisation par des non spécialistes d'informatique. Nous envisageons aussi le déploiement de GATE sur une grille de supercalculateurs dans le cadre du projet européen DEISA, faisant ainsi de GATE la seule application commune aux deux gros projets d'infrastructure de grille en Europe.

Remerciements. Le projet EGEE-II est financé par la Commission européenne sur le contrat $n^{\circ}$ INFSO-RI-031688. Le cluster du Biopôle Clermont-Limagne a été financé par le Conseil Général. Le projet Auvergrid est financé par le Conseil Régional. Nous remercions le Centre de Calculs de l'IN2P3 pour la mise à 
disposition de ressources. Nous remercions l'équipe de Roberto Barbera (Université de Catagne - INFN) pour son soutien dans l'utilisation du portail GENIUS ainsi que le service informatique du LPC pour l'assistance apportée dans l'utilisation des ressources informatiques du laboratoire.

\section{RÉFÉRENCES}

Adronico A., Barbera R., Falzone A., Kunszt P., Lo Re G., Pulvirenti A., Rodolico A. (2003) GENIUS: a simple and easy way to access computational and data grids, Future Generation Computer Systems 19(6), 805-813.

Bramoullé C. (2000) Etude par le code MC PENELOPE des faisceaux de rayons X produits par des accélérateurs d'électrons SL-ELEKTA, Thèse, Université Paul Sabatier.

Douglas J., Simpkin T., Rockwell M. (1990) EGS4 Monte-Carlo determination of the beta dose kernel in water, Med. Phys. 17(2), 179-186.

Hedtjarn H., Carlsson G., Williamson J.F. (2000) Monte Carlo-aided dosimetry of the symmetra model I25.S06 ${ }^{125} \mathrm{I}$, interstitial brachytherapy seed, Med. Phys. 27(5).

Hokkanen J., Heikkonen J., Holmberg P. (1997) Theorical calculations of dose distributions for betaray eye applicators, Med. Phys. $\mathbf{2 4}(2)$.

Jan S. et al. (2004) GATE: a simulation toolkit for PET and SPECT, Phys. Med. Biol. 49, 4543-4561.

Lazaro D. (2003) Validation de la plate-forme de simulation GATE en Tomographie d'Emission Monophotonique et application au développement d'un algorithme de reconstruction 3D complète, Thèse, Université Blaise Pascal, PCCF T 0304.

Maigne L. (2005) Personalized dosimetry using GATE Monte Carlo simulations on grid architecture: Application in ocular brachytherapy, Thése, Université Blaise Pascal, PCCF T 0508.

Maigne L., Hill D., Calvat P., Breton V., Reuillon R., Lazaro D., Legré Y., Donnarieix D. (2004) Parallelization of Monte Carlo Simulations and Submission to a Grid Environment, Parallel Proc. Lett. J. 14(2), 177-196.

Masurier J. (1999) Adaptation du code MC PENELOPE pour la métrologie de la dose absorbée : caractérisation des faisceaux de photons $X$ et calcul de facteurs de correction de dosimètres de référence, Thèse, Université Paul Sabatier.

Mobit P., Badragan I. (2004) An evaluation of the AAPM-TG43 dosimetry protocol for I-125 brachytherapy seed, Phys. Med. Biol. 49, 3161-3170.

Nath R., Anderson L.L., Luxton G., Weaver K.A., Williamson J.F., Meigooni A.S. (1995) Dosimetry of interstitial brachytherapy sources: Recommendations of the AAPM Radiation Therapy Committee Task Group No. 43, Med. Phys. 22(2), 209-234.

Soares C.G., Vynckier S., Järvinen H., Cross W.G., Sipilä P., Flühs D., Schaeken B., Mourtada F.A., Bass G.A., Williams T.T. (2001) Dosimetry of beta-ray ophthalmic applicators: Comparison of different measurements methods, Med. Phys. 28(7), 1373-1384.

Tjho-Heslinga R.E., Davelaar J., Kemme H.M., De Vroome H., Oosterhuis J.A., Bleeker J.C., Leer J.W.H. (1999) Results of ruthenium irradiation of uveal melanomas: the Dutch experience, Radiotherapy and Oncology 53, 133-137. 